\title{
LES POROHALACARIDAE DE LA FAUNE FRANÇAISE
}

par E. ANGelier.

Les Halacariens d'eau douce sont encore peu connus en France. A. Migot [1926] décrivit le premier; c'était une nouvelle espèce, - Lobohalacarus gallicus. K. VIErs [1939] signala ensuite la présence de Soldanellonyx chappuisi Walter dans une source du lac d'Artouste (Basses-Pyrénées), à l'altitude de $1864 \mathrm{~m}$. Des recherches poursuivies de 1947 à 1952 , tant par le $\mathrm{D}^{\mathrm{r}}$ P.-A. Chappurs que par moi-même, sur la faune des eaux superficielles et souterraines, ont permis de recueillir 4 autres espèces, dont une, - Porolohmannella andrei, - nouvelle pour la Science [E. ANGELIER, 1951, 1952].

En 1959, M. J.-Y. PiCARD me confiait une collection de Porohalacaridae récoltés dans les eaux interstitielles de Lorraine; une espèce était nouvelle pour la faune française, - Soldanellonyx monardi.

Enfin, des prospections dans quelques lacs de haute altitude des Pyrénées centrales m'ont permis de recueillir 4 espèces, dont une, - Porohalacarus hydrachnoüdes, — était encore inconnue en France.

Les Porohalacaridae sont donc représentées actuellement dans notre pays par 8 espèces :

\section{1. - Porohalacarus hydrachnoïdes (LohmanN 1893) (fig. 1-2)}

Décrit par Lohmann [1893], Porohalacarus hydrachnoüdes est resté longtemps une espèce mal connue, confondue parfois avec $P$. alpinus. K. ViETs [1938] en a donné une nouvelle description, et, plus récemment, Ramazzotti et Nocentini [1960] et NocenTrNI [1961] l'ont retrouvée dans le lac de Mergozzo et dans le lac Majeur, en talie.

$P$. hydrachnoïdes est nouveau pour la faune française; il vit sur les fonds de vase des lacs de l'Estibère (Hautes-Pyrénées), entre 2100 et $2300 \mathrm{~m}$ d'altitude (coll. E. Angelier).

Les individus recueillis ont une longueur de $300 \mu$ environ (ㅇ). La plaque dorsale atteint les $2 / 3$ de la longueur du corps $(210 \mu)$; clle est très étroite $(56 \mu)$. Les plaques oculaires sont réduites.

Les cupules génitales sont en nombre variable (6 à 8 en général). 
Longueur, en $\mu$, des articles des pattes (mesurés sur le bord extenseur) :

\begin{tabular}{|c|c|c|c|c|c|c|}
\hline & I & II & III & IV & V & VI \\
\hline p 1 & 25 & 14 & 58 & 34 & 65 & 41 \\
\hline p 2 & 22 & 12 & 48 & 32 & 53 & 39 \\
\hline p 3 & 34 & 14 & 41 & 25 & 51 & 54 \\
\hline p 4 & 34 & 14 & 34 & 31 & 31 & 60 \\
\hline
\end{tabular}

Les griffes, à l'extrémité du $6^{\text {" }}$ article, sont simples. Le 5 article des pattes 1 porte 2 paires d'épines lisses sur le bord fléchisseur et 3 soies sur le bord extenseur. Sur les pattes 2 à 4 , le $5^{\text {e }}$ article ne présente que 3 épines ( 2 distales et 1 médiane). Le nombre d'épines est le même sur le $4^{\mathrm{e}}$ article de toutes les pattes : 2, sur le bord fléchisseur.

Cotte espèce n'a jamais été recueillie dans les eaux interstitielles; elle paraît caractéristique des fonds de vase, dans les eaux lacustres.

\section{2. - Lobohalacarus weberi (RomiJn \& VIETs 1924) (fig. 3)}

\section{Stations françaises connues :}

Coll. E. Angelier. - Ruisseau de l'Oursière, dans le massif de Belledonne (Isère). — Tech, près d'Elne (Pyrénées-Orientales). Têt, dans le Massif du Carlitte (Pyrénées-Orientales). - Rivière Porto, près de son embouchure (Corse). - Rivière Aitone, sous le col de Vergio (Corse). — Lacs de l'Estibère; Neste de Couplan (Hautes-Pyrénées).

\section{3. - Lobohalacarus weberi quadriporus (WALTER 1947)}

Cette sous-espèce est caractéristique des eaux interstitielles, alors que l'espèce s. str., beaucoup plus ubiquiste, se rencontre aussi bien dans les mousses des eaux courantes que dans les eaux interstitielles ou lacustres.

Stations françaises connues :

Coll. E. Angelier. - Fier, près d'Annecy (Haute-Savoie) et près de son confluent avec le Rhône. - Tech, à la Preste (Pyrénées- 
(3)

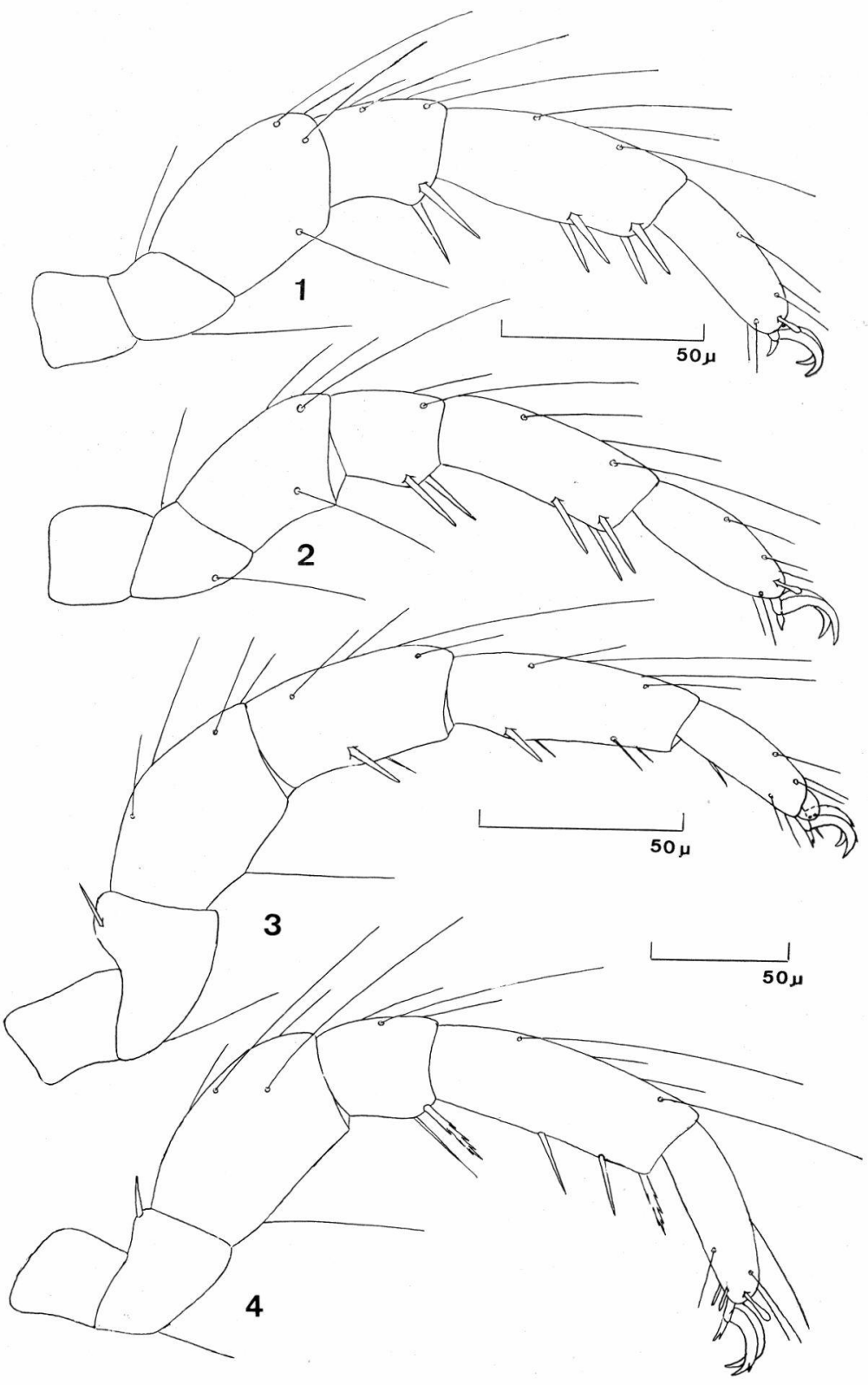

FIg. 1 : Porohalaca us hydrachnö̈des, patte 1. - FIG. 2 : Porohalacarus hydrachnoüdes, patte 2. - FIG. 3 : Lobahalacarus weberi, patte 1. Fig. 4. : Lobohalacarus gallicus, patte 1. 
Orientales). - Brèbre, affluent rive gauche de la Loire, à Jaligny (Allier).

Coll. J.-Y. Picard. - Ruisseau de Sarre Blanche, affluent de la Sarre. - Ruisseaux de Plaine, de Vezouze, de Mortagne, affluents de la Meurthe. - Meurthe, Moselotte, Moselle (Lorraine).

\section{4. - Lobohalacarus gallicus (MIGot 1925) (fig. 4) \\ Syn. : L. dolgarae GREEN 1954.}

Le type de Lobohalacarus gallicus a été récolté dans un étang du Massif du Carlitte (Pyrénées-Orientales). Il a été retrouvé ensuite, soit par le $\mathrm{D}^{\mathbf{r}}$ P.-A. Chappuis, soit par moi-même, en d'autres points des Pyrénées.

J. Green [1954] a décrit une nouvelle espèce, L. dolgarae, d'Angleterre. L'auteur signale, comme caractère essentiel différenciant L. dolgarae et $L$. gallicus, la présence de 3 soies, — au lieu de 2, - sur le $4^{\text {e }}$ article des palpes maxillaires. Or, ces 3 soies existent aussi chez L. gallicus; la fig. donnée in E. ANGElier 1952 n'en montre que 2 par erreur. Ces 3 soies constituent d'ailleurs un caractère générique, et non spécifique. Les corps et pattes des 2 espèces étant identiques, $L$. dolgarae est à mon avis synonyme de $L$. gallicus.

Stations françaises connues :

Coll. A. Migot. - Vase sur le fond d'un étang du « désert du Carlitte 》(Pyrénées-Orientales), à $2200 \mathrm{~m}$ d'altitude environ.

Coll. P.-A. Chappuis. - Grotte de l'Église, à Nistos (Ariège).

Coll. F. Angelier. - Psammon du ruisseau de Grave, en amont du lac des Bouillouses (Pyrénées-Orientales).

Fait intéressant : alors que le type de Lobohalacarus gallicus a été récolté sur des vases lacustres de haute altitude dans les Pyrénées-Orientales, c'est $L$. weberi que j'ai toujours recueilli dans les lacs de l'Estibère (Hautes-Pyrénées) à des altitudes identiques.

\section{5. - Soldanellonyx chappuisi WaLter 1917 (fig. 5)}

Stations françaises connues :

Coll. K. Viets. - Source près du lac d'Artouste (Basses-Pyrénées).

Coll. P.-A. Chappuis. - Aven Sainte-Catherine (Ariège. - Hount Santo (Ariège).

Coll. J.-Y. Picard. - Graviers sur le bord de la Moselle et du ruisseau de Mortagne (affluent de la Meurthe) (Lorraine). 


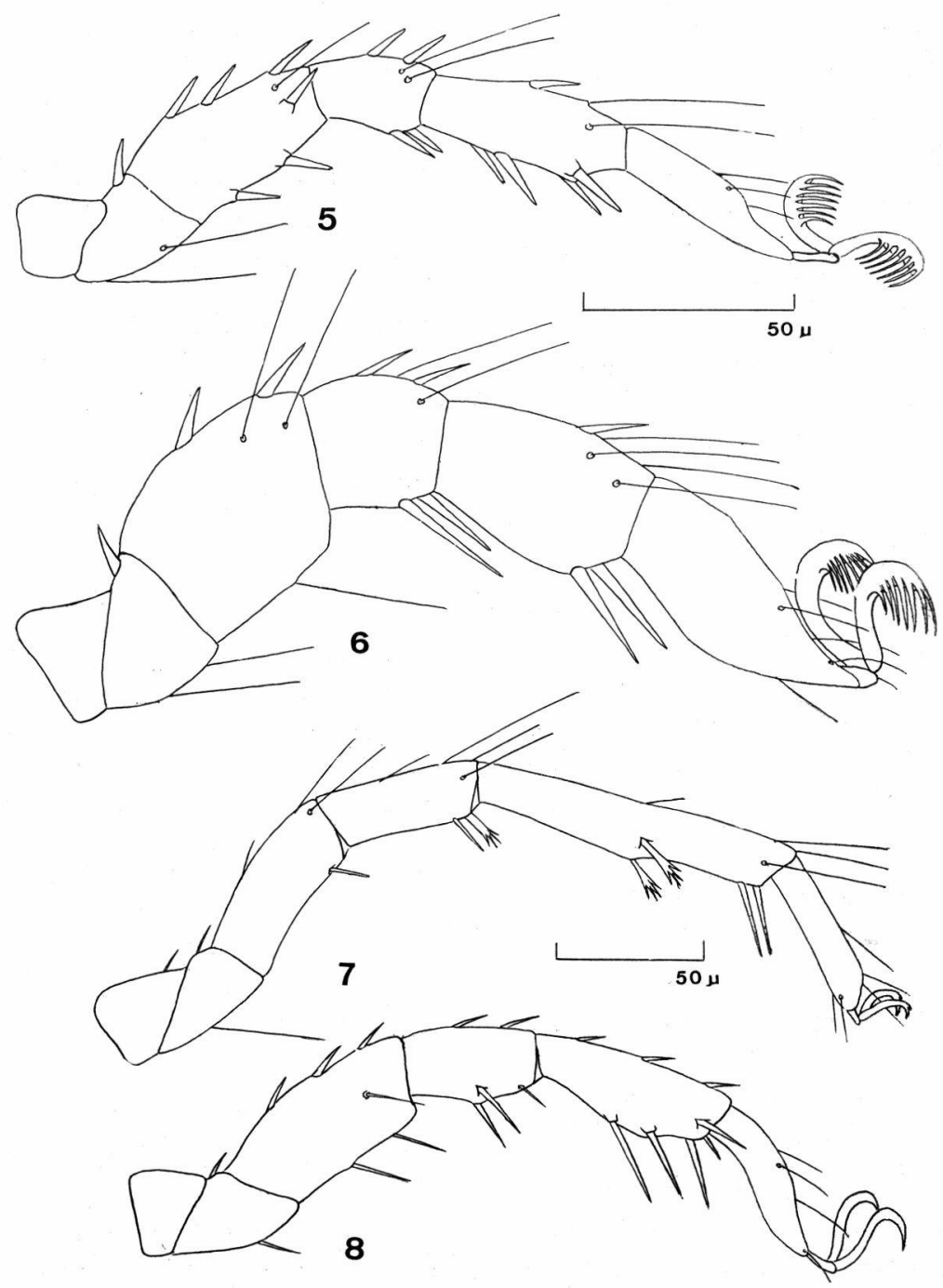

FIG. 5 : Soldan'ellonyx chappuisi, patte 1. - Fig. 6 : Soldanellonyx monardi, patte 1. - Fig. 7 : Porolohmannella violacea, patte 1. - Fig. 8 : Porolohmannella andrei, patte 1 . 


\section{6. -- Soldanellonyx monardi WaLTER 1919 (fig. 6)}

Cette espèce est nouvelle pour la faune française. Les exemplaires de Lorraine, comme ceux des Pyrénées, sont tout-à-fait semblables au type. La longueur du corps varie entre 350 et $370 \mu$; celle de la plaque dorsale atteint $280 \mu$ en moyenne. Le nombre des cupules génitales est de 6 à 8 de chaque côté.

Longueur, en $\mu$, des articles des pattes (mesurés sur le bord extenseur) :

\begin{tabular}{|c|c|c|c|c|c|c|}
\hline & I & II & III & IV & $\mathrm{V}$ & VI \\
\hline p 1 & 31 & 10 & 48 & 33 & 51 & 61 \\
\hline p 2 & 29 & 8 & 46 & 34 & 51 & 58 \\
\hline p 3 & 34 & 20 & 40 & 34 & 54 & 64 \\
\hline p 4 & 37 & 20 & 44 & 36 & 56 & 66 \\
\hline
\end{tabular}

Les pattes sont caractérisées par des griffes portant chacune un peigne sur le bord interne et une dent accessoire sur le bord externe. Le $5^{\text {e }}$ article présente sur toutes 2 longues épines, - l'une lisse, l'autre pennée, - à l'extrémité distale du bord fléchisseur. De plus, le $5^{*}$ article des pattes 1 porte une courte épine lisse sur le bord extenseur; le $5^{\text {e }}$ article des pattes 2 en porte 2.

Stations françaises connues :

Coll. F. Angelier. - Lacs de l'Estibère (Hautes-Pyrénées).

Coll. J.-Y. Picard. - Ruisseaux de Châtillon et de Marquis, se jetant dans le Vezouze, affluent de la Meurthe (Lorraine).

\section{3. - Porolohmanne la violacea (KRAMER 1879) (fig. 7)}

Siations françaises connues :

Coll. E. Angelier. - Lacs de l'Estibère (Hautes-Pyrénées). - Vase sur le fond du lac de Nino et graviers sur le bord du Tavignano, près de sa source (Corse).

\section{8. - Porolohmannela andrei E. Angelier 1951 (fig. 8)}

Par les caractères du palpe maxillaire et des pattes, cette espèce se rattache plus à Lohmannella falcata HodGE et Lohmannella stammeri Viets qu'à Porolohmannella violacea. L. stammeri, décrite par Viets [1939 c] d'une grotte de l'Italie du Sud située à 
$200 \mathrm{~m}$ de la mer, est une forme cavernicole appartenant à un genre marin. L. falcata, en Méditerranée, est une forme très psammophila. $P$. andrei, qui vit dans le milieu interstitiel des PyrénéesOrientales, est peut-être une relicte marine au même titre que nombre de Crustacées recueillis avec lui .

Stations françaises connues :

Coll. E. Angelier. - Baillaurie, près de Banyuls-sur-mer (Pyrénées-Orientales). — Tech, à Prats-de-Mollo (Pyrénées-Orientales).

\section{The Porohalacaridae of the French fauna}

Only 6 species of Porohalacaridae were known in France. Research in Lorraine and the central Pyrenees have permitted the discovery of 2 species new to France.

\section{Die Porohalacaridae aus der französische Fauna}

Nur 6 Arten der Porohalacaridae waren in Frankreich bekannt. Die Forschungen die in den Zentralpyrenäen und in Lorraine durchgeführt worden sind, führten zur Entdeckung von 2 neuen Arten für die franzözische Fauna.

\section{TRAVAUX CITÉS}

Angelier (E.). 1951. - Porolohmannella andrei n. sp., un nouvel Halacarien recueilli dans le psammon d'eau douce. Bull. Mus. Hist. Paris, 23, 5 : 505-507.

ANGelier (E.). 1952. - Note sur Lobohalacarus gallicus (Migot 1926) et les Porohalacaridae (Acari) de la faune française. Bull. Mus. Nat. Hist. Nat. Paris, 24, 2 : 195-198.

Green (J.). 1954. - A new species of Lobohalacarus an other Porohalacarids (Acari) in Windermere. Proc. Zool. Soc. London, 124, $3: 669-674$.

Lohmann (H. T.). 1893. - Die Halacarinen der Plankton-Expedition. In V. Hensen, Ergebnisse der in den Atlantischen Ocean von Mitte Juli bis Anfang November 1889 ausgeführten Plankton-Expedition der Humboldt-Stiftung. Kiel \& Leipzig, 2 : 11-95.

Migot (A.-M.). 1961. - Le $1^{\text {er }}$ Halacaride de la faune française, Walterella gallica n. sp. Bull. Soc. Zool. Fr., 51 : 376-383.

Nocentini (A. M.). 1961. - Primi ritrovamenti di Porohalacaridae (Acari) nel Lago Maggiore. Mem. Ist. Ital. Idrobiol., 13 : 127-138. 
PiCard (J. Y.). 1962. - Contribution à la connaissance de la faune psammique de Lorraine. Vie et Milieu, 12, $3: 471-505$.

Ramazzotti (G.) et Nocentini (A. M.). 1960. - Porohalacaridae (Acarina) del Lago di Mergozzo. Mem. Ist. Ital. Idrobiol., 12 : 185-200.

VieTs (K.). 1938. - Ueber Porohalacarus hydrachnoides (Lohmann 1893). Z6ol. Anz., 122, 1/2 : 45-48.

Viets (K.). 1939 a. - Wassermilben aus französischen Pyrenäen. Zool. Anz., 125, $1: 1-15$.

Viets (K.). 1939 b. - Ueber die Milbengruppe der Porohalacaridae (Acari). Zugleich ein Beitrag zur Kenntnis der Milbenfauna der Bremen Umgegend und des Plöner Sees. Abh. naturw. Ver. Bremen, 31, $3: 502-514$.

VIETs (K.). 1939 c. - Halacariden aus süditalienischen Höhlengewässern. Arch. Hydrobiol., 36, 4 : 625-630.

Viets (K.). 1956. - Die Milben des Süsswassers und des Meeres. Vol. 2, 870 p. G. Fischer Verl., Jéna.

(Laboratoire de Zoologie de la Faculté des Sciences de Toulouse.) 